\title{
Association of the roles of advanced glycation end products and osteocalcin between bone metabolism and vascular failure
}

\author{
Ippei Kanazawa, $\mathrm{MD}, \mathrm{PhD}$
}

\begin{abstract}
:
Fragility fracture impairs the activities of daily living and quality of life of the elderly. Accumulating evidence has shown that patients with osteoporosis have an increased all-cause mortality as well as cardiovascular mortality rates. Osteoporosis and cardiovascular diseases have common risk factors, such as diabetes mellitus. Patients with diabetes have an increased risk of osteoporosis; thus, diabetes-related bone disease is now recognized as one of the complications of diabetes. Although accumulation of advanced glycation end products and oxidative stress are associated with the formation and progression of atherosclerosis, these are reported to be involved in the pathogenesis of osteoporosis, especially in diabetic patients. Moreover, recent studies have shown that osteocalcin, which is secreted from the bone into the circulation, has an endocrine function of regulating glucose and energy metabolism. In addition, osteocalcin directly affects vascular endothelial cells and smooth muscle cells and protects against oxidative stress-induced cell dysfunction. Therefore, the bone-vascular axis attracts widespread attention. In this review, I described the association between bone and glucose metabolism and vascular failure on the basis of recent evidence.
\end{abstract}

Key words:

Bone, Osteoporosis, Osteocalcin, Advanced glycation end product, Atherosclerosis

\section{Introduction}

Both osteoporosis and cardiovascular disease (CVD) are important health problems worldwide because they deteriorate the quality of life and mortality of the patients. These diseases have been traditionally viewed as separate entities that increase in prevalence with aging. However, accumulating evidence has shown that they may be associated with each other. A previous large-scale cohort study showed that each decrease of standard deviation in bone mineral content was associated with a $43 \%$ increase in mortality in postmenopausal women ${ }^{1)}$. When only cardiovascular death was considered, the relative risk of dying within $17 \mathrm{y}$ after menopause increased to 2.3 -fold. In addition, women with bone mass in the lowest quartile had twice the risk of cardiovascular death compared with those in the highest quartile, and a prevalent vertebral fracture was independently associated with cardiovascular death with a 2.2 -fold increase. Observation of postmenopausal women who received a placebo treatment in an osteoporosis treatment trial showed that subjects with osteoporosis had a 3.9-fold increased risk for CVD events compared with those with osteopenia ${ }^{2}$. Moreover, subjects with vertebral fracture also had an increased risk of CVD events up to 3.0-fold compared with those without vertebral fractures, and the risk of CVD events increased incrementally as the number and severity of baseline vertebral fractures also increased. Furthermore, several clinical studies using bone mineral density (BMD) and parameters of atherosclerosis have been reported to date. BMD at the femoral neck was significantly and inversely correlated with common carotid artery intima-media thickness (IMT) and pulse wave velocity (PWV) in postmenopausal women with osteoporosis ${ }^{3}$. Moreover, a similar tendency in healthy subjects independent of common risk factors for osteoporosis and CVD was found ${ }^{4,5}$. A longitudinal study showed that a decrease in BMD was significantly associated with increased aortic calcification scores ${ }^{6}$. Additionally, we previously reported that the presence of osteoporosis was a risk factor for increased IMT and aortic calcification in postmenopausal women with type 2 diabetes mellitus (T2DM) .

Internal Medicine 1, Shimane University Faculty of Medicine

Corresponding author: Ippei Kanazawa, MD, PhD, ippei.k@med.shimane-u.ac.jp

Received: January 19, 2017, Accepted: March 30, 2017

Copyright (C) 2017 Japan Society for Vascular Failure 
These findings indicate that osteoporosis accompanies CVD and that these 2 diseases may be associated with each other.

Although the mechanism is not fully understood, there are common exacerbation factors of osteoporosis and CVD. Recently, advanced glycation end products (AGEs) were shown to play a pivotal role in atherosclerotic diseases and osteoporosis. Conversely, the bone has been recognized as an endocrine organ. Osteocalcin is expressed and produced specifically in the osteoblasts and has an endocrine function of regulating glucose and energy homeostasis ${ }^{8}$. Moreover, osteocalcin may be associated with CVD risk. In this review, I summarized the role of AGEs as a common factor of CVD and osteoporosis and the role of osteocalcin in the interaction between the bone and CVD on the basis of the above mentioned recent evidence.

\section{AGEs as a common factor of CVD and osteoporosis}

There are several common factors of osteoporosis and CVD, including aging, smoking, alcohol abuse, lack of exercise, and menopause. Lifestyle-related diseases, such as diabetes, dyslipidemia, and chronic kidney disease are well known to increase CVD risk. These lifestyle-related diseases also increase osteoporotic fracture risk that may be mainly caused by a deteriorated bone quality". AGEs are generated via the sequential nonenzymatic chemical glycoxidation of protein amino groups. AGEs accumulate in various tissues, including the bone, kidney, brain, and coronary artery with aging. Thus, AGE formation is considered as a cumulative metabolic stress marker, adversely influencing the aging process and chronic disease development and progression across the life course. Further, AGEs have a pivotal role in the development of complications in patients with diabetes, because hyperglycemia and oxidative stress accelerate AGE formation.

Blood AGE concentration is associated with CVD risk and mortality ${ }^{10,11}$. AGEs are also involved in the atherosclerotic process and vascular failure. AGEs increase intracellular oxidative stress generation and inflammation-related gene expression in vascular wall cells. AGEs inhibit endothelial nitric oxide (NO) synthase (eNOS) expression and NO production $^{12,13)}$, which is the most potent endogenous vasodilator and antiatherogenic factor. Moreover, AGEs impair endothelial cell repair, enhance apoptosis, and suppress the migration and tube formation of endothelial progenitor cells via Akt and cycloxygenase-2 inhibition ${ }^{14,15}$. AGEs also contribute to vascular calcification, the severity of which is known to be a predictor of CVD events and mortality. AGEs induce the osteoblastic differentiation and mineralization of pericytes $^{16)}$, which have the plasticity to differentiate into smooth muscle cells. Furthermore, AGEs increase oxidative stress by stimulating NADPH oxidase (Nox) expression and induce vascular smooth muscle cell apoptosis and osteoblastic transdifferentiation $^{17,18)}$ (Figure 1). Several types of AGEbinding proteins have been identified ${ }^{19}$. Among them, the re- ceptor for AGE (RAGE) is reported to be a cell surface receptor that belongs to the immunoglobulin superfamily and plays an important role in the action of AGEs. Indeed, in diabetic ApoE knockout model mice, the deletion of RAGE significantly reduced the atherosclerotic plaque area, attenuated leukocyte recruitment, decreased proinflammatory mediator expression, including that of nuclear factor- $\mathrm{kB}$ (NF$\kappa \mathrm{B}), \mathrm{VCAM}-1$, and MCP-1, and reduced oxidative stress and AGE accumulation in plaques ${ }^{20)}$. AGE-RAGE signaling elicits oxidative stress generation and evokes inflammatory and thrombogenic reactions ${ }^{21}$. In addition, AGEs increase RAGE expression and induce sustained NF- $\kappa B$ activation ${ }^{22}$, which is located on the RAGE promoter, linking RAGE to inflammation. Thus, a vicious cycle exists, in which AGERAGE signaling induces oxidative stress, subsequently enhancing further generation and accumulation of AGEs and RAGE overexpression.

Conversely, AGE-RAGE signaling is involved in the pathogenesis of osteoporosis, especially in bone quality deterioration. Although the underlying mechanism of bone quality deterioration is largely unknown, accumulation of AGEs in the bone matrix is considered an important cause $\mathrm{e}^{23}$. Among AGEs, pentosidine is a well-characterized compound and is considered a good predictor for micro- and macrovascular complication development in diabetic patients. Serum pentosidine levels in patients with diabetes were significantly higher than those in healthy subjects. Further, spontaneous diabetic rats displayed significantly increased pentosidine cross-links in the bone, which was linked to impaired mechanical properties despite a normal bone mass ${ }^{23}$. As circulating pentosidine levels are significantly correlated with the pentosidine content in the cortical bone, serum and urine pentosidine levels could be used as surrogate markers for its content in the bone and bone strength. Indeed, elevated serum and urine pentosidine levels were significantly associated with fracture risks in elderly patients with $\mathrm{T}_{2} \mathrm{DM}^{24,25)}$. In addition, a recent clinical study using bone biopsy in patients with T1DM showed that pentosidine content in the trabecula was significantly and positively associated with HbA1c levels and increased in patients with T1DM with fracture $^{26}$. Therefore, pentosidine cross-link accumulation in the bone may be the major cause of impaired bone quality in patients with diabetes.

\section{AGEs induce osteoblast and osteocyte dysfunction}

The bone tissue is constantly renewed by a balance between osteoblastic bone formation and osteoclastic bone resorption. Bone formation markers, especially serum osteocalcin, which is a marker of bone formation and produced by mature osteoblasts, significantly decreased in patients with diabetes compared to non-diabetic subjects ${ }^{27)}$. We previously demonstrated that serum osteocalcin levels significantly increased after intensive glycemic control in T2DM, while bone-specific alkaline phosphatase (BAP), 


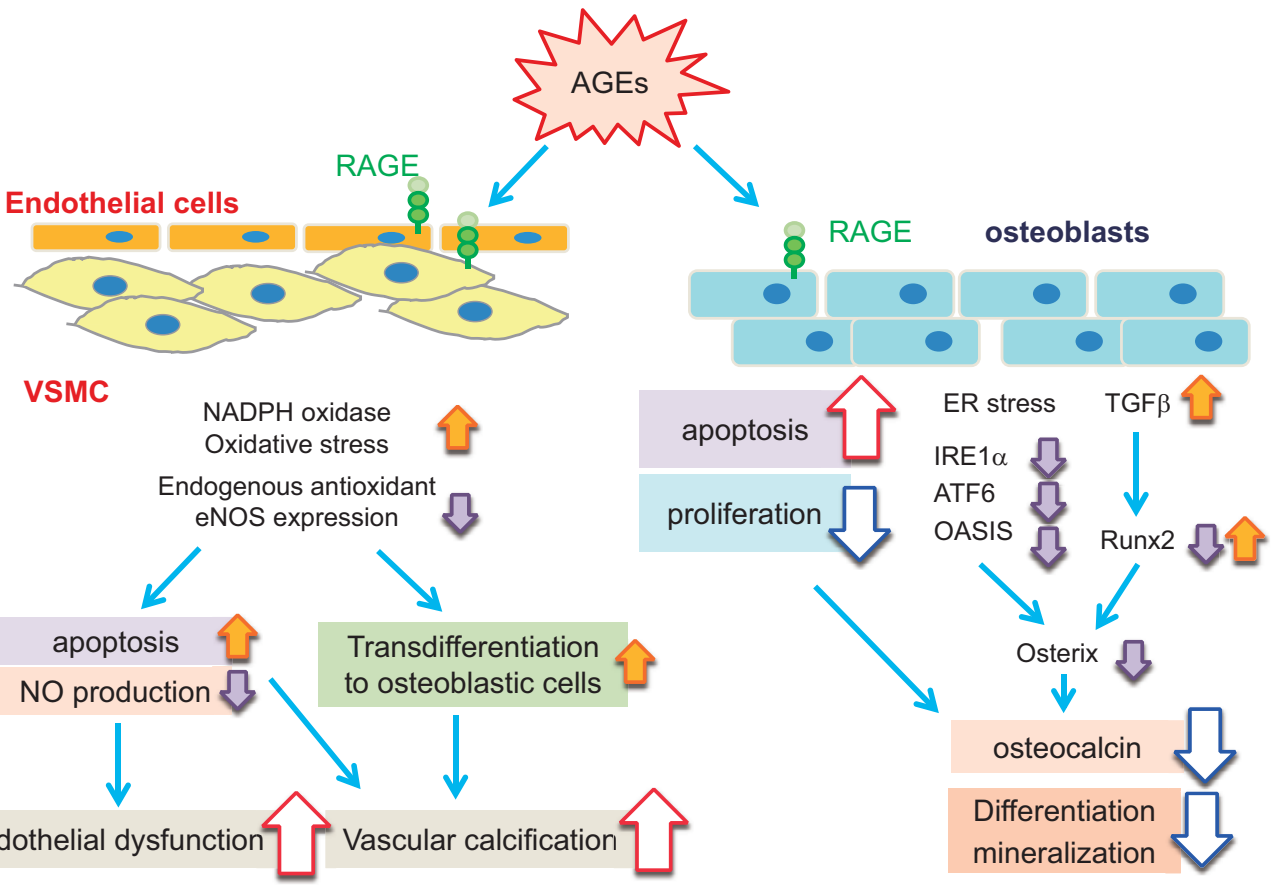

Figure 1. Direct effects of advanced glycation end products (AGEs) on vascular cells and osteoblasts.

The receptor for AGEs (RAGE) is expressed in the endothelial cells and vascular smooth muscle cells (VSMC), and AGEs act as physiological molecules. AGE-RAGE signaling enhances NADPH oxidase expression and increases oxidative stress but inhibits the expression of endogenous antioxidants and production of endothelial nitric oxide (NO) synthase (eNOS). AGEs induce apoptosis of endothelial cells and VSMC and inhibit NO production in the endothelial cells. Moreover, AGEs induce transdifferentiation of VSMC to osteoblastic cells. Thus, AGE-RAGE signaling induces endothelial dysfunction and vascular calcification. Conversely, RAGE is also expressed in osteoblasts. AGEs induce apoptosis and suppress cell growth of osteoblasts. AGEs inhibit the differentiation and mineralization of osteoblasts through endoplasmic reticulum (ER) stress and TGF $\beta$ expression, thereby decreasing bone formation.

which is a marker of the early stage of differentiated osteoblasts, significantly decreased ${ }^{28)}$. Moreover, the osteocalcin/BAP ratio was significantly associated with prevalent vertebral fractures in patients with $\mathrm{T} 2 \mathrm{DM}^{29}$. These findings suggest that osteoblast maturation derangement may be associated with fracture risks in diabetic patients. Conversely, osteocytes account for $90-95 \%$ of bone cells and play multifunctional roles in orchestrating bone remodeling by regulating both osteoblast and osteoclast functions. Sclerostin is specifically expressed in osteocytes and inhibits osteoblast function and bone formation by antagonizing the canonical Wnt signaling pathway. Because elevated serum sclerostin levels were associated with increased vertebral fracture risks in patients with T2DM independent of BMD and bone turnover $^{30)}$, osteocyte dysfunction may also contribute to bone fragility.

As RAGE is expressed in osteoblasts and osteocytes, there is a possibility that AGEs directly affect bone formation and remodeling. The combination of high glucose and AGEs inhibited osteocalcin expression and osteoblastic cell line, MC3T3-E1, mineralization ${ }^{31}$; further, AGEs inhibited the osteoblastic differentiation or mineralization of mouse stromal ST2 cells and human mesenchymal stem cells by decreasing osterix expression, increasing transforming growth factor (TGF)- $\beta$ expression, and suppressing endoplasmic reticulum (ER) stress proteins ${ }^{32,33)}$ (Figure 1). Moreover, high glucose and AGEs significantly increased sclerostin expression in osteocyte-like MLO-Y4 cells ${ }^{34)}$. In contrast, AGEs decreased the receptor activator of NF- $\mathrm{kB}$ ligand (RANKL) expression in the cells and induced apoptosis of osteoblasts and osteocytes. In summary, AGEs directly inhibit osteoblastic differentiation and bone formation and indirectly by increasing sclerostin expression in osteocytes, as well as contribute to low turnover of bone remodeling by decreasing RANKL expression.

\section{Glucose metabolism regulation by osteocalcin}

Osteocalcin, which is one of the osteoblast-specific proteins and has several hormonal features, regulates glucose metabolism ${ }^{8)}$. Osteocalcin knockout $\left(\mathrm{Ocn}^{-1-}\right)$ mice were previously generated to investigate the roles of osteocalcin in the bone tissues ${ }^{35)}$. Although it was not reported at that time, the $\mathrm{Ocn}^{-1-}$ mice were obese and had an abnormal visceral fat accumulation. In 2007, it was reported that $\mathrm{Och}^{-/-}$mice 
displayed hyperglycemia and glucose intolerance due to insulin insufficiency and resistance ${ }^{8}$. In these mice, pancreatic $\beta$-cell proliferation and insulin secretion significantly decreased, and insulin resistance from a decreased adiponectin expression in adipocytes was observed. Moreover, when Ocn expression vector-transfected COS cells were cocultured with islets or adipocytes, the insulin and adiponectin expression was significantly enhanced.

Osteocalcin has 46-50 amino acids and undergoes $\gamma$ carboxylation of glutamyl residues at 3 positions ${ }^{17,21,24)}$, which facilitates the binding of osteocalcin to hydroxyapatite in the bone matrix. Further examinations have shown that an undercarboxylated form of osteocalcin (ucOC) is an active form in glucose metabolism ${ }^{8,36}$. Esp encodes osteotesticular protein tyrosine phosphatase (OST-PTP), which is restricted to osteoblasts, sertoli cells, and embryonic stem cells ${ }^{37}$. OST-PTP is a transmembrane tyrosine phosphatase, which cannot directly affect distant tissues. Because OST-PTP stimulates carboxylation of osteocalcin and decreases osteocalcin bioactivity, $\mathrm{Esp}^{-/-}$mice were examined as a model of gain of osteocalcin bioactivity ${ }^{8)}$. In contrast to $\mathrm{Och}^{-/-}$ mice, $E_{s p^{-/-}}$mice showed hypoglycemia and low blood glucose levels after glucose injection, increased insulin expression and secretion, increased insulin sensitivity, and increased adiponectin expression in the adipose tissues. Furthermore, $E_{s p^{-1-}}$ mice displayed decreased fat mass and serum triglyceride levels and resistance to high-fat dietinduced obesity and diabetes and to streptozotocin-induced diabetes. The metabolic phenotype of $\mathrm{Ocn}^{-1-}$ mice is the mirror image of that seen in $E s p^{-/-}$mice. To examine whether the metabolic abnormalities in $E s p^{-1-}$ mice could be corrected by the inhibition of osteocalcin expression, Esp ${ }^{-1-}$ mice were crossed with $\mathrm{Ocn}^{+/-}$mice. In $\mathrm{Esp}^{-/-}: \mathrm{Ocn}^{+/-}$ mice, the metabolic abnormalities, such as hypoglycemia, hyperinsulinemia, and increased serum adiponectin level were completely reversed.

In addition to the direct effect of osteocalcin on insulin secretion, osteocalcin indirectly stimulates insulin expression and secretion by increasing the secretion of glucagon-like peptide-1 (GLP-1), which is an incretin released by intestinal endocrine cells. Mizokami et al. demonstrated that the treatment with ucOC significantly increased GLP-1 expression in STC-1 enteroendocrine cells in vitro and that the administration of ucOC increased serum GLP-1 and insulin levels in mice ${ }^{38,39}$. These effects were potentiated by an inhibitor of dipeptidyl peptidase-4 and blocked by a GLP-1 receptor antagonist, suggesting that ucOC increases insulin secretion by enhancing GLP-1 secretion from intestinal endocrine cells.

The effects of recombinant osteocalcin injection on glucose metabolism in wild-type (WT) mice were previously reported $^{36)}$. Continuous intraperitoneal injection of low dose recombinant osteocalcin increased insulin secretion, pancreatic $\beta$-cell proliferation, insulin sensitivity, and adiponectin expression and decreased fat mass in WT mice. Moreover, recombinant osteocalcin injection prevented high-fat diet- induced obesity, fatty liver, and glucose intolerance. Further, therapeutic potential intermittent administration of recombinant osteocalcin was also tested ${ }^{40)}$. Daily injection of osteocalcin significantly improved glucose intolerance and insulin resistance in mice fed not only with normal diet but also with a high-fat diet. In addition, hepatic steatosis induced by a high-fat diet was completely recovered in mice treated with daily osteocalcin injection. Interestingly, the oral administration of osteocalcin also improved impaired glucose tolerance in vivo ${ }^{38,39,41}$. The ucOC via oral administration reached the small intestines, remained there for at least $24 \mathrm{~h}$, and entered the general circulation. Daily and long-term intermittent oral administration of ucOC significantly reduced fasting blood glucose level and improved glucose tolerance in mice without affecting insulin sensitivity. Oral administration also increased fasting serum insulin level and $\beta$-cell area in the pancreas. The serum GLP-1 level was increased in accordance with the presence of ucOC in the intestines and systemic circulation. In summary, these findings suggest that the intermittent injection and oral administration of recombinant osteocalcin may be useful in treating T2DM and obesity.

Previous studies suggest that the G-protein-coupled receptor family $\mathrm{C}$ group 6 member A (GPRC6A) is a receptor for osteocalcin and mediates the response to osteocalcin in $\beta$ cells $^{42}$. GPRC6A is an orphan receptor belonging to the Gprotein-coupled receptors, which are known as seventransmembrane domain receptors, and is ubiquitously expressed and sense amino acids and extracellular calcium ${ }^{43,44}$. It is reported that GPRC6A knockout mice showed osteopenia, hyperglycemia, impaired glucose tolerance, insulin resistance, and hepatic steatosis ${ }^{45}$, suggesting that GPRC6A may participate in the anabolic response of multiple tissues. On the basis of the metabolic abnormalities in the GPRC6A knockout mice, Pi et al. ${ }^{44}$ hypothesized that GPRC6A might be involved in the function of ucOC in glucose homeostasis. To investigate the role of GPRC6A in osteocalcin function, the effects of osteocalcin on GPRC6A-expressed cells were investigated. Recombinant osteocalcin stimulated ERK activity in HEK-293 cells overexpressing GPRC6A in a dosedependent manner but did not affect the untransfected control cells. It was confirmed that the pancreatic $\beta$-cell TC- 6 cell line and pancreas isolated from WT mice expressed GPRC6A and that the recombinant osteocalcin treatment stimulated ERK activity in vitro and in vivo. Moreover, administration of recombinant osteocalcin significantly increased insulin expression in the pancreas as well as serum insulin levels in the WT mice but not in the GPRC6A knockout mice.

\section{Effects of osteocalcin on vascular cells and atherosclerosis}

As described above, osteocalcin regulates glucose and energy homeostasis (Figure 2). In addition, adiponectin and GLP-1 are known to have favorable effects on the formation 


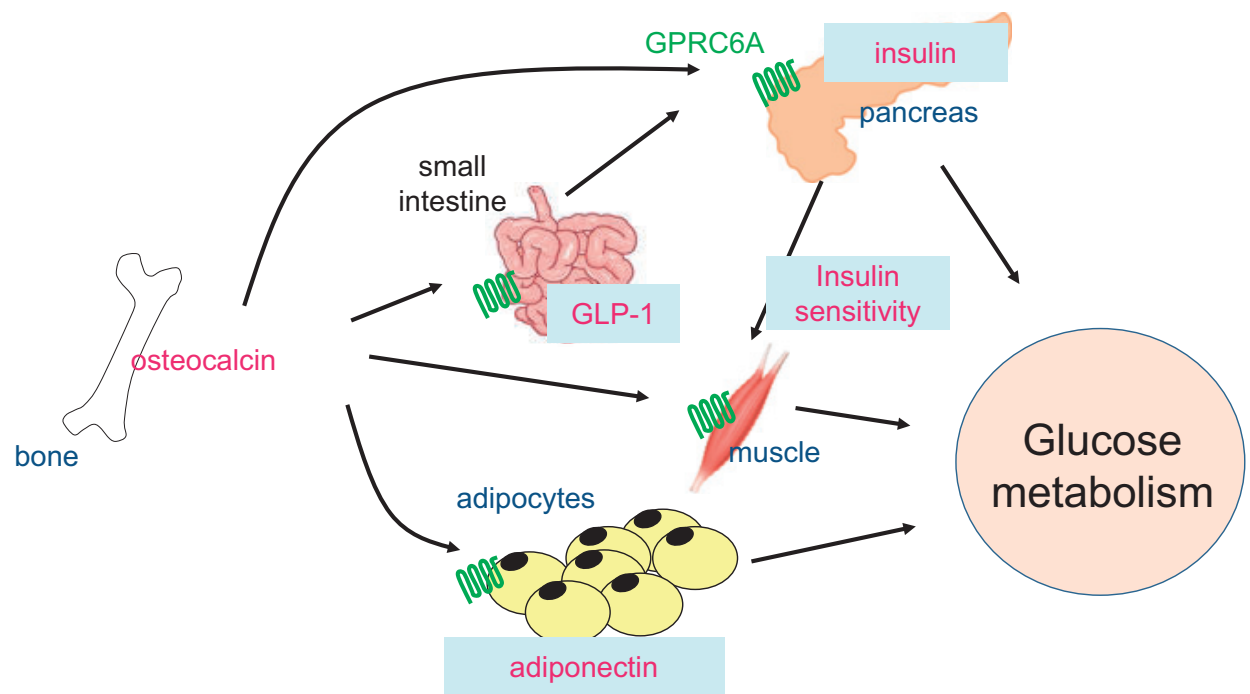

Figure 2. Schematic representation of the mechanisms regulating glucose metabolism by the bone.

Osteocalcin secreted from the bone directly stimulates insulin secretion from the pancreas and indirectly by increasing the secretion of glucagon-like peptide-1 (GLP-1) from the small intestines. Osteocalcin stimulates the expression of adiponectin in the adipose tissues, thereby increasing insulin sensitivity. Osteocalcin also enhances insulin signaling in the muscles. G-protein-coupled receptor family C group 6 member A (GPRC6A) is a receptor for undercarboxylated osteocalcin (ucOC) and is expressed in pancreatic $\beta$-cells, epithelial cells of the small intestines, adipocytes, and myotubes.

and progression of atherosclerosis. The evidence suggests that osteocalcin may indirectly affect CVD. However, several studies have shown that osteocalcin might directly affect the blood vessels because the receptor for osteocalcin, GPRC6A, exists on endothelial cells and vascular smooth muscle cells ${ }^{46)}$. A previous in vitro study showed that osteocalcin prevented free fatty acid-induced apoptosis of vascular endothelial cells ${ }^{47}$. Moreover, osteocalcin enhanced the expression of eNOS and secretion of NO by activating the phosphatidylinositol 3-kinase and Akt signaling pathways. In addition, the daily injection of osteocalcin suppressed the abnormal autophagy and ER stress induced by a high-fat diet in the vascular tissue ${ }^{48}$. A high-fat diet induced autophagy, increased autophagic indicators, such as Atg7 and LC3-II, decreased p62, and activated ER stress sensor PERK and its downstream molecule, eIF2alpha. Osteocalcin injection improved these effects of high-fat diet on the abnormal autophagy and ER stress. Moreover, they showed similar results by using the cell lines of vascular endothelial cells and vascular smooth muscle cells. The role of osteocalcin in vascular function has been reported ${ }^{49)}$. Daily injections of osteocalcin can significantly improve lipid metabolism, glucose tolerance, and insulin sensitivity in ApoE-deficient mice. In ApoE-deficient mice fed with a high-fat diet, the osteocalcin-treated mice displayed an improved acetylcholinestimulated endothelium-dependent relaxation (EDR) compared with the vehicle-treated mice. Moreover, osteocalcintreated human umbilical vein endothelial cells displayed increased activation of the Akt-eNOS signaling pathway compared with the vehicle-treated cells. Furthermore, a similar beneficial effect of osteocalcin on the thoracic aorta was observed using an ex vivo organ culture of an isolated mouse aortic segment. These findings suggest that osteocalcin has beneficial effects against metabolic stress-induced vascular dysfunction by stimulating eNOS expression and regulating ER stress in the vascular tissues (Figure 3).

Since in vitro and in vivo studies have shown that osteocalcin plays crucial roles in glucose metabolism and has protective effects on metabolic stress-induced vascular dysfunction, of particular interest is whether osteocalcin level in the circulation is associated with atherosclerosis parameters and CVD events in humans. Indeed, the size and some amino acids of osteocalcin are different between mice and humans, and osteocalcin is encoded by a single gene in humans that is highly conserved across species, while mice contain a cluster of 3 osteocalcin genes ${ }^{44}$. We have previously shown for the first time that serum osteocalcin levels were significantly and inversely associated with IMT and PWV in men with T2DM independent of conventional risk factors for $\mathrm{CVD}^{50)}$. In addition, we found a negative association between changes in serum osteocalcin and plaque score during glycemic control in patients with T2DM in a longitudinal study ${ }^{51}$. Since then, several clinical studies on the association between osteocalcin and atherosclerosis and CVD have been reported. Some studies demonstrated that serum osteocalcin levels were inversely associated with atherosclerotic parameters and artery calcification ${ }^{52-59)}$; however, other studies reported otherwise ${ }^{60,61)}$. Sheng et al. ${ }^{52)}$ showed that serum osteocalcin levels were significantly and inversely associated with IMT and carotid plaques in men with T2DM via 


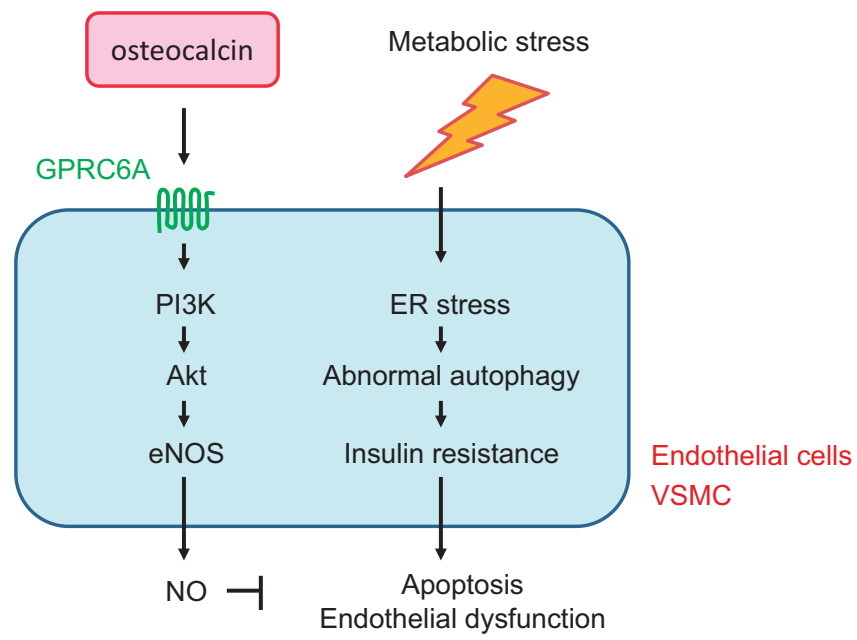

Figure 3. Protective effects of osteocalcin against metabolic stress-induced vascular failure.

Metabolic stress, such as hyperglycemia and obesity, increases endoplasmic reticulum (ER) stress and induces abnormal autophagy and insulin resistance in endothelial cells and vascular smooth muscle cells (VSMC), resulting in apoptosis of the cells and endothelial dysfunction. In contrast, osteocalcin activates phosphatidylinositol 3-kinase (PI3K) /Akt signaling and increases endothelial nitric oxide (NO) synthase (eNOS) expression. Osteocalcin has a protective effect against vascular failure induced by metabolic stress. a multiple adjustment analysis. Yang et al. ${ }^{53)}$ conducted a further large-scale cross-sectional study and showed that serum osteocalcin levels were negatively associated with IMT in 1,319 Chinese postmenopausal women without any history of CVD or carotid plaque. Kim et al. ${ }^{54)}$ reported that serum osteocalcin showed an age-independent association with aortic calcification scores. We also demonstrated that decreased serum osteocalcin and ucOC levels were significantly associated with increased aortic calcification scores in men with $\mathrm{T}_{2} \mathrm{DM}^{55)}$. Moreover, Choi et al. ${ }^{56)}$ examined the coronary artery calcium score (CACS) by using a multidetector computed tomography and showed an independent negative association between serum ucOC and CACS in men without angina symptoms. In a prospective cohort study of community-dwelling men aged 70-89 y, the highest and lowest quintiles of serum osteocalcin showed a significant increase in all-cause and cardiovascular mortality rates ${ }^{57)}$, suggesting a U-shaped association of serum osteocalcin with such rates in men with high risks of CVD. In contrast, Zhang et al. ${ }^{58)}$ reported a linear relation between serum osteocalcin level and coronary artery disease (CAD) in 461 subjects who underwent coronary angiography. Serum osteocalcin levels were significantly lower in patients with CAD than in non-CAD subjects and significantly decreased as the number of diseased vessels increased. Bao et al. ${ }^{59)}$ also investigated 181 men who underwent coronary angiography and reported that osteocalcin was independently associated with waist circumference and plasma glucose level as well as the presence of metabolic syndrome. In addition, a

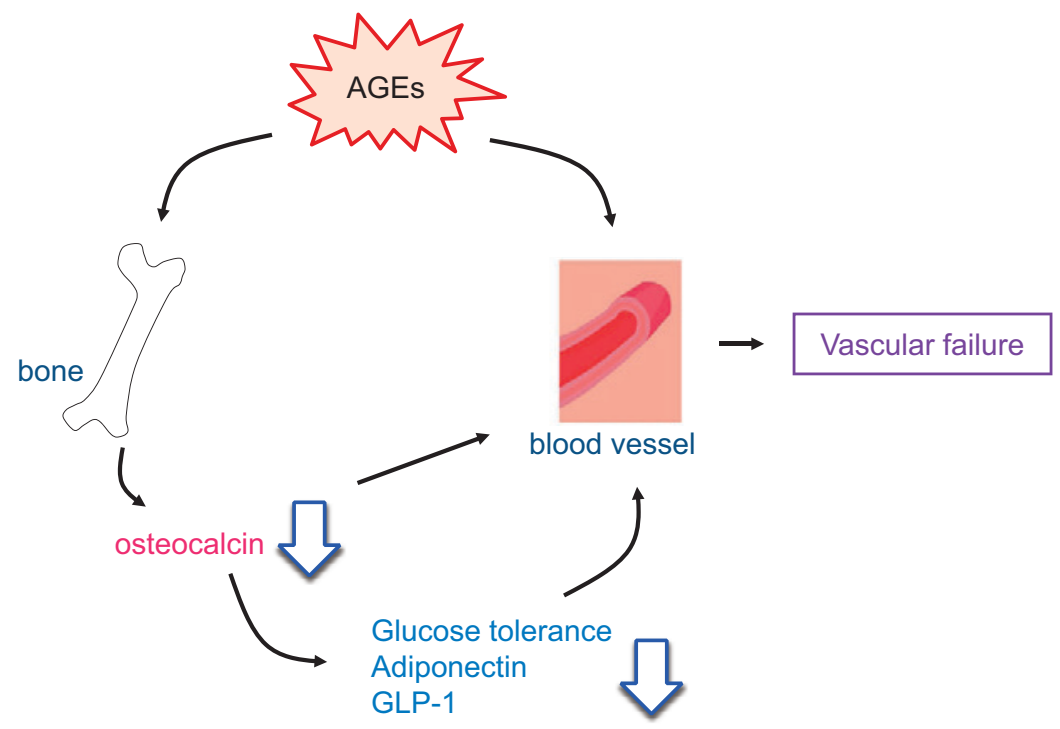

Figure 4. Schematic representation of the association between the bone and vascular failure.

Advanced glycation end products (AGEs) have direct harmful effects on bone metabolism and vascular cells. AGEs inhibit osteoblastic differentiation and osteocalcin secretion from the bone. AGE-suppressed osteocalcin expression induces glucose intolerance and decreases expression of adiponectin and glucagon-like peptide-1 (GLP-1), both of which have beneficial effects on vascular function. Thus, AGEs are directly and indirectly involved in the mechanisms of vascular failure. 
subgroup analysis of subjects with normal glucose tolerance showed that serum osteocalcin significantly decreased in patients with coronary artery disease compared with those without and as the number of stenotic vessels increased.

Taken together, these findings suggest that osteocalcin derived from the bone plays pivotal roles in the association between osteoporosis and CVD. Osteocalcin may have direct effects on atherosclerosis and CVD events as well as indirect effects by regulating glucose and fat metabolism. However, the number of studies on osteocalcin and CVD is limited. In addition, it is still controversial whether ucOC is the active form of the endocrine factor in humans. Therefore, further large-scale studies and meta-analyses are necessary in the future.

\section{Summary}

In summary, it has been shown that osteoporosis and CVD are associated with each other. AGEs are involved in the pathology of osteoporosis and CVD; thus, these can be a candidate therapeutic target for both diseases. There are 2 different strategies of anti-AGE signaling. The first is to inhibit either the production and accumulation of AGEs or the signaling pathway at the level of their receptors. The second is to increase the breakdown of already existing AGEs in the atherosclerotic lesion and bone matrix. Although several existing drugs, such as statins and metformin, are reported to reduce the formation of AGEs and inhibit their signaling ${ }^{62}$, further pre-clinical and clinical studies are necessary. Moreover, the bone secretes osteocalcin into the circulation, which regulates glucose and fat metabolism and directly affects the formation of atherosclerosis. Since AGEs inhibit osteocalcin expression in osteoblasts, AGEs indirectly deteriorate vascular function by affecting the interaction of the bone and vascular tissue (Figure 4). Therefore, in cases of osteoporosis and CVD, it may be beneficial to enhance the expression of osteocalcin and bone formation.

Conflicts of Interest

None.

\section{References}

1. von der Recke P, Hansen MA, Hassager C. The association between low bone mass at the menopause and cardiovascular mortality. Am J Med 1999; 106: 273-8.

2. Tanko LB, Christiansen C, Cox DA, Geiger MJ, McNabb MA, Cummings SR. Relationship between osteoporosis and cardiovascular disease in postmenopausal women. J Bone Miner Res 2005; 20: 1912-20.

3. Okamoto K, Inaba M, Furumitsu Y, Ban A, Mori N, Yukioka K, et al. Beneficial effect of risedronate on arterial thickening and stiffening with a reciprocal relationship to its effect on bone mass in female osteoporosis patients: a longitudinal study. Life Sci 2010; 87: 686-91.

4. Yamada S, Inaba M, Goto $H$, Nagata-Sakurai M, Kumeda Y, Imanishi $Y$, et al. Associations between physical activity, peripheral atherosclerosis and bone status in healthy Japanese women. Athe- rosclerosis 2006; 188: 196-202.

5. Hirose K, Tomiyama H, Okazaki R, Arai T, Koji Y, Zaydun G, et al. Increased pulse wave velocity associated with reduced calcaneal quantitative osteo-sono index: possible relationship between atherosclerosis and osteopenia. J Clin Endocrinol Metab 2003; 88: 2573-8.

6. Kiel DP, Kauppila LI, Cupples LA, Hannan MT, O’Donnell CJ, Wilson PW. Bone loss and the progression of abdominal aortic calcification over a 25 year period: the Framingham Heart Study. Calcif Tissue Int 2001; 68: 271-6.

7. Kanazawa I, Yamaguchi T, Hayashi K, Takase H, Shimizu T, Sugimoto T. Effects of treatment with risedronate and alfacalcidol on progression of atherosclerosis in postmenopausal women with type 2 diabetes mellitus accompanied with osteoporosis. Am J Med Sci 2010; 339: 519-24.

8. Lee NK, Sowa H, Hinoi E, Ferron M, Ahn JD, Confavreux C, et al. Endocrine regulation of energy metabolism by the skeleton. Cell 2007; 130: 456-69.

9. Vestergaard P. Discrepancies in bone mineral density and fracture risk in patients with type 1 and type 2 diabetes--a meta-analysis. Osteoporos Int 2007; 18: 427-44.

10. Wagner Z, Molnar M, Molnar GA, Tamasko M, Laczy B, Wagne L, et al. Serum carboxymethyllysine predicts mortality in hemodialysis patients. Am J Kidney Dis 2006; 47: 294-300.

11. Furuya R, Kumagai H, Miyata T, Fukasawa H, Isobe $S$, Kinoshita $\mathrm{N}$, et al. High plasma pentosidine level is accompanied with cardiovascular events in hemodialysis patients. Clin Exp Nephrol 2012; 16: 421-6.

12. Soro-Paavonen A, Zhang WZ, Venardos K, Coughlan MT, Harris $\mathrm{E}$, Tong DC, et al. Advanced glycation end-products induce vascular dysfunction via resistance to nitric oxide and suppression of endothelial nitric oxide synthase. J Hypertens 2010; 28: 780-8.

13. Ando R, Ueda S, Yamagishi S, Miyazaki H, Kaida Y, Kaifu K, et al. Involvement of advanced glycation end product-induced asymmetric dimethylarginine generation in endothelial dysfunction. Diab Vasc Dis Res 2013; 10: 436-41.

14. Chen Q, Dong L, Wang L, Kang L, Xu B. Advanced glycation end products impair function of late endothelial progenitor cells through effects on protein kinase Akt and cyclooxyge-nase-2. Biochem Biophys Res Commun 2009; 381: 192-7.

15. Bhatwadekar AD, Glenn JV, Li G, Curtis TM, Gardiner TA, Stitt AW. Advanced glycation of fibronectin impairs vascular repair by endothelial progenitor cells: implications for vasodegeneration in diabetic retinopathy. Invest Ophthalmol Vis Sci 2008; 49: 1232-41.

16. Yamagishi $S$, Imaizumi T. Pericyte biology and diseases. Int J Tissue React 2005; 27: 125-35.

17. Tada Y, Yano S, Yamaguchi T, Okazaki K, Ogawa N, Morita M, et al. Advanced glycation end products-induced vascular calcification is mediated by oxidative stress: functional roles of $\mathrm{NAD}(\mathrm{P}) \mathrm{H}-$ oxidase. Horm Metab Res 2013; 45: 267-72.

18. Koike S, Yano S, Tanaka S, Sheikh AM, Nagai A, Sugimoto T. Advanced glycation end-products induce apoptosis of vascular smooth muscle cells: a mechanism for vascular calcification. Int $\mathrm{J}$ Mol Sci 2016; 17: E1567.

19. Sourris $\mathrm{KC}$, Forbes JM. Interactions between advanced glycation end-products (AGE) and their receptors in the development and progression of diabetic nephropathy: are these receptors valid therapeutic targets. Curr Drug Targets 2009; 10: 42-50.

20. Soro-Paavonen A, Watson AM, Li J, Paavonen K, Koitka A, Calkin AC, et al. Receptor for advanced glycation end products (RAGE) deficiency attenuates the development of atherosclerosis in diabetes. Diabetes 2008; 57: 2461-9.

21. Yamagishi S, Nakamura N, Suematsu M, Kaseda K, Matsui T. Ad- 
vanced glycation end products: a molecular target for vascular complications in diabetes. Mol Med 2015; 21 Suppl 1: S32-40.

22. Xie J, Mendez JD, Mendez-Valenzuela V, Aguilar-Hernandez MM. Cellular signaling of the receptor for advanced glycation end products (RAGE). Cell Signal 2013; 25: 2185-97.

23. Saito M, Fujii K, Mori Y, Marumo K. Role of collagen enzymatic and glycation induced cross-links as a determinant of bone quality in spontaneously diabetic WBN/Kob rats. Osteoporos Int 2006; 17: 1514-23.

24. Yamamoto M, Yamaguchi T, Yamauchi M, Yano S, Sugimoto T. Serum pentosidine levels are positively associated with the presence of vertebral fractures in postmenopausal women with type 2 diabetes. J Clin Endocrinol Metab 2008; 93: 1013-9.

25. Schwartz AV, Garnero P, Hillier TA, Sellmeyer DE, Strotmeyer ES, Feingold KR, et al. Health, aging, and body composition study. J Clin Endocrinol Metab 2009; 94: 2380-6.

26. Farlay D, Armas LA, Gineyts E, Akhter MP, Recker RR, Boivin G. Nonenzymatic glycation and degree of mineralization are higher in bone from fractured patients with type 1 diabetes mellitus. J Bone Miner Res 2016; 31: 190-5.

27. Liu C, Wo J, Zhao Q, Wang Y, Wang B, Zhao W. Association between serum total osteocalcin level and type 2 diabetes mellitus: a systematic review and meta-analysis. Horm Metab Res 2015; 47: 813-9.

28. Kanazawa I, Yamaguchi T, Yamauchi M, Yamamoto M, Kurioka S, Yano S, et al. Adiponectin is associated with changes in bone markers during glycemic control in type 2 diabetes mellitus. J Clin Endocrinol Metab 2009; 94: 3031-7.

29. Kanazawa I, Yamaguchi T, Yamamoto M, Yamauchi M, Yano S, Sugimoto T. Serum osteocalcin/bone-specific alkaline phosphatase ratio is a predictor for the presence of vertebral fractures in men with type 2 diabetes. Calcif Tissue Int 2009; 85: 228-34.

30. Yamamoto M, Yamauchi M, Sugimoto T. Elevated sclerostin levels are associated with vertebral fractures in patients with type 2 diabetes mellitus. J Clin Endocrinol Metab 2013; 98: 4030-7.

31. Ogawa N, Yamaguchi T, Yano S, Yamauchi M, Yamamoto M, Sugimoto T. The combination of high glucose and advanced glycation end-products (AGEs) inhibits the mineralization of osteoblastic MC3T3-E1 cells through glucose-induced increase in the receptor for AGEs. Horm Metab Res 2007; 39: 871-5.

32. Notsu M, Yamaguchi T, Okazaki K, Tanaka K, Ogawa N, Kanazawa I, et al. Advanced glycation end product 3 (AGE3) suppresses the mineralization of mouse stromal ST2 cells and human mesenchymal stem cells by increasing TGF- $\beta$ expression and secretion. Endocrinology 2014; 155: 2402-10.

33. Tanaka K, Yamaguchi T, Kaji H, Kanazawa I, Sugimoto T. Advanced glycation end products suppress osteoblastic differentiation of stromal cells by activation endoplasmic reticulum stress. Biochem Biophys Res Commun 2013; 438: 463-7.

34. Tanaka K, Yamaguchi T, Kanazawa I, Sugimoto T. Effects of high glucose and advanced glycation end products on the expressions of sclerostin and RANKL as well as apoptosis in osteocyte-like MLO-Y4-A2 cells. Biochem Biophys Res Commun 2015; 461: 193-9.

35. Ducy P, Desbois C, Boyce B, Pinero G, Story B, Dunstan C, et al. Increased bone formation in osteocalcin-deficient mice. Nature 1996; 382: 448-52.

36. Ferron M, Hinoi E, Karsenty G, Ducy P. Osteocalcin differentially regulates beta cell and adipocyte gene expression and affects the development of metabolic diseases in wild-type mice. Proc Natl Acad Sci USA 2008; 105: 5266-70.

37. Mauro LJ, Olmsted EA, Skrobacz BM, Mourey RJ, Davis AR, Dixon JE. Identification of a hormonally regulated protein tyrosine phosphatase associated with bone and testicular differentiation. J Biol Chem 1994; 269: 30659-67.

38. Mizokami A, Yasutake Y, Gao J, Matsuda M, Takahashi I, Takeuchi $\mathrm{H}$, et al. Osteocalcin induces release of glucagon-like peptide- 1 and thereby stimulates insulin secretion in mice. PLoS One 2013; 8: e57375.

39. Mizokami A, Yasutake Y, Higashi S, Kawakubo-Yasukochi T, Chishaki S, Takahashi I, et al. Oral administration of osteocalcin improves glucose utilization by stimulating glucagon-like peptide1 secretion. Bone 2014; 69: 68-79.

40. Ferron M, McKee MD, Levine RL, Ducy P, Karsenty G. Intermittent injections of osteocalcin improve glucose metabolism and prevent type 2 diabetes in mice. Bone 2012; 50: 568-75.

41. Otani T, Mizokami A, Hayashi Y, Gao J, Mori Y, Nakamura S, et al. Signaling pathway for adiponectin expression in adipocytes by osteocalcin. Cell Signal 2015; 27: 532-44.

42. Kuang D, Yao Y, Lam J, Tsushima RG, Hampson DR. Cloning and characterization of a family $\mathrm{C}$ orphan G-protein coupled receptor. J Neurochem 2005; 93: 383-91.

43. Wellendorph $\mathrm{P}$, Brauner-Osborne $\mathrm{H}$. Molecular cloning, expression, and sequence analysis of GPRC6A, a novel family C Gprotein-coupled receptor. Gene 2004; 335: 37-46.

44. Pi M, Faber P, Ekema G, Jackson PD, Ting A, Wang N, et al. Identification of a novel extracellular cation-sensing G-proteincoupled receptor. J Biol Chem 2005; 280: 40201-9.

45. Desbois C, Hogue DA, Karsenty G. The mouse osteocalcin gene cluster contains three genes with two separate spatial and temporal patterns of expression. J Biol Chem 1994; 269: 1183-90.

46. Harno E, Edwards G, Geraghty AR, Ward DT, Dodd RH, Dauban $\mathrm{P}$, et al. Evidence for the presence of GPRC6A receptors in rat mesenteric arteries. Cell Calcium 2008; 44: 210-9.

47. Jung CH, Lee WJ, Hwang JY, Lee MJ, Seol SM, Kim YM, et al. The preventive effect of uncarboxylated osteocalcin against free fatty acid-induced endothelial apoptosis through the activation of phosphatidylinositol 3-kinase/Akt signaling pathway. Metabolism 2013; 62: 1250-7.

48. Zhou B, Li H, Liu J, Xu L, Zang W, Wu S, et al. Intermittent injections of osteocalcin reverse autophagic dysfunction and endoplasmic reticulum stress resulting from diet-induced obesity in the vascular tissue via the NFKB-p65-dependent mechanism. Cell Cycle 2013; 12: 1901-13.

49. Dou J, Li H, Ma X, Zhang M, Fang Q, Nie M, et al. Osteocalcin attenuates high fat diet-induced impairment of endotheliumdependent relaxation through Akt/eNOS-dependent pathway. Cardiovasc Diabetol 2014; 13: 74.

50. Kanazawa I, Yamaguchi T, Yamamoto M, Yamauchi M, Kurioka S, Yano S, et al. Serum osteocalcin level is associated with glucose metabolism and atherosclerosis parameters in patients with type 2 diabetes. J Clin Endocrinol Metab 2009; 94: 45-9.

51. Kanazawa I, Yamaguchi $T$, Sugimoto $T$. Relationship between bone biochemical markers versus glucose/lipid metabolism and atherosclerosis: a longitudinal study in type 2 diabetes mellitus. Diabetes Res Clin Pract 2011; 92: 393-9.

52. Sheng L, Cao W, Cha B, Chen Z, Wang F, Liu J. Serum osteocalcin level and its association with carotid atherosclerosis in patients with type 2 diabetes. Cardiovasc Diabetol 2013; 12: 22.

53. Yang R, Ma X, Dou J, Wang F, Luo Y, Li D, et al. Relationship between serum osteocalcin levels and carotid intima-media thickness in Chinese postmenopausal women. Menopause 2013; 20: 1194-9.

54. Kim KJ, Kim KM, Park KH, Choi HS, Rhee Y, Lee YH, et al. Aortic calcification and bone metabolism: the relationship between aortic calcification, BMD, vertebral fracture, 25-hydroxyvitamin 
D, and osteocalcin. Calcif Tissue Int 2012; 91: 370-8.

55. Ogawa-Furuya N, Yamaguchi T, Yamamoto M, Kanazawa I, Sugimoto T. Serum osteocalcin levels are inversely associated with abdominal aortic calcification in men with type 2 diabetes mellitus. Osteoporos Int 2013; 24: 2223-30.

56. Choi BH, Joo NS, Kim MJ, Kim KM, Park KC, Kim YS. Coronary artery calcification is associated with high serum concentration of undercarboxylated osteocalcin in asymptomatic Korean men. Clin Endocrinol 2015; 83: 320-6.

57. Yeap BB, Chubb SA, Flicker L, McCaul KA, Ebeling PR, Hankey GJ, et al. Associations of total osteocalcin with all-cause and cardiovascular mortality in older men. The Health in Men Study. Osteoporos Int 2012; 23: 599-606.

58. Zhang Y, Qi L, Gu W, Yan Q, Dai M, Shi J, et al. Relation of serum osteocalcin level to risk of coronary heart disease in Chinese adults. Am J Cardiol 2010; 106: 1461-5.
59. Bao Y, Zhou M, Lu Z, Li H, Wang Y, Sun L, et al. Serum levels of osteocalcin are inversely associated with the metabolic syndrome and the severity of coronary artery disease in Chinese men. Clin Endocrinol 2011; 75: 196-201.

60. Luo Y, Ma X, Hao Y, Xiong Q, Xu Y, Pan X, et al. Relationship between serum osteocalcin level and carotid intima-media thickness in a metabolically healthy Chinese population. Cardiovasc Diabetol 2015; 14: 82.

61. Kang JH. Association of serum osteocalcin with insulin resistance and coronary atherosclerosis. J Bone Metab 2016; 23: 183-90.

62. Nenna A, Spadaccio C, Lusini M, Ulianich L, Chello M, Nappi F. Basic and clinical research against advanced glycation end products (AGEs): new compounds to tackle cardiovascular disease and diabetic complications. Recent Adv Cardiovasc Drug Discov 2015; 10: $10-33$. 